\title{
Real-time increase in depth of field of an uncooled thermal camera using several phase-mask technologies
}

\author{
Frédéric Diaz, ${ }^{1,3, *}$ Mane-Si Laure Lee, ${ }^{1}$ Xavier Rejeaunier, ${ }^{2}$ Gaelle Lehoucq, ${ }^{1}$ François Goudail, \\ Brigitte Loiseaux, ${ }^{1}$ Shailendra Bansropun, ${ }^{1}$ Joel Rollin, ${ }^{2}$ Eric Debes, ${ }^{1}$ and Philippe Mils ${ }^{1}$ \\ ${ }^{1}$ Thales Research \& Technology, 1 Avenue Augustin Fresnel, 91767 Palaiseau Cedex, France \\ ${ }^{2}$ Thales Angénieux, Boulevard Ravel de Malval, 42750 Saint-Héand, France \\ ${ }^{3}$ Laboratoire Charles Fabry de l'Institut d'Optique, CNRS, Université Paris-Sud, \\ Campus Polytechnique, RD 128, 91127 Palaiseau Cedex, France \\ *Corresponding author: frederic.diaz@thalesgroup.com
}

Received October 29, 2010; revised January 4, 2011; accepted January 5, 2011; posted January 7, 2011 (Doc. ID 137364); published February 1, 2011

\begin{abstract}
Imaging systems that combine a phase mask in the pupil and digital postprocessing may have better performance than conventional ones. We have built such a system to enhance the depth of field of an uncooled thermal camera. The phase masks are binary, their structures are optimized thanks to an image quality criterion, and they have been realized with three different technologies that give equivalent results. The deconvolution postprocessing is performed in real time with a graphics processing unit. A significant increase of the depth of field of a factor 3 has been obtained. (c) 2011 Optical Society of America
\end{abstract}

OCIS codes: $110.7348,110.3080,050.6624,100.1830$.

In traditional imaging systems, the purpose of the optics is to provide an image as sharp as possible. The ability of digital postprocessing to correct some aberrations of the optics is not taken into account while designing such a system. Conversely, in a hybrid imaging approach, the optics and the postprocessing step are designed together. Using this principle, Cathey and Dowski [1] proposed to combine a cubic phase mask that makes the point-spread function of the optical system insensitive in a large range of defocus, with digital deconvolution to recover the image quality. Thanks to an optimization criterion that we introduced in a previous Letter [2] and based on the image quality after the deconvolution, we are able to optimize the shape of the cubic phase mask and of any other type of masks [3], according to the characteristics of the optics, the noise of the sensor, and the targeted range of defocus.

Our goal is to validate experimentally this optimization criterion by increasing by a factor 3 the depth of field of an uncooled bolometric camera with an $F$ number equal to 1.3 , a focal length of $18 \mathrm{~mm}$, a pixel size of $25 \mu \mathrm{m}$, and operating in the long wave IR $(8-12 \mu \mathrm{m})$. The defocus of the camera is quantified by the following parameter:

$$
\psi=\pi R^{2} / \lambda \times\left(1 / d_{O}+1 / d_{I}-1 / f\right),
$$

where $R$ is the radius of the aperture, $\lambda$ is the wavelength, and $d_{O}, d_{I}$, and $f$ are the object distance, the image sensor plane distance, and the focal length, respectively. A conventional imaging system provides sharp images for $\psi=[-2.5 ; 2.5]$.

For our purpose, among the different types of phase masks, we chose to implement the binary-phase mask (BPM) [4], as it is the easiest to manufacture. The BPM is composed of an array of three concentric rings whose phases are alternatively zero and $\pi$ [Fig. 1(a)]. Their outer radii are denoted $r_{1}$ and $r_{2}$ and are determined as a result of the optimization criterion of [2], in order to keep the image quality for an extended range of defocus $\psi \in$ $[-7.6 ; 7.6]$. The image obtained with the optical system is

$$
I(r)=h_{\psi}(r) * O(r)+n(r),
$$

where the symbol $*$ refers to the convolution operation, $O(r)$ is the object, $n(r)$ is the measure noise, and $h_{\psi}(r)$ is the point-spread function of the optical system at a given defocus $\psi$. Then the image is deconvolved with the Wiener-like filter $d$, described in [2], resulting in an estimate of the object $\hat{O}_{\psi}(r)$ expressed as

$$
\hat{O}_{\psi}(r)=d(r) * I(r) .
$$

The mean-square error $\mathrm{MSE}_{\psi}$ between the estimate and the true object is

$$
\mathrm{MSE}_{\psi}=\left\langle\left|\hat{O}_{\psi}(r)-O(r)\right|^{2}\right\rangle .
$$

The image-quality criterion is then given by

$$
\mathrm{SNR}_{\text {out }}=10 \log _{10}\left[\int S_{o o}(\nu) \mathrm{d} \nu / \min _{\psi}\left(\mathrm{MSE}_{\psi}\right)\right] \text {, }
$$

where $S_{O O}(\nu)$ is the power spectrum density of the object.

We have represented in Fig. $1(\mathrm{~b})$ the $\mathrm{SNR}_{\text {out }}$ (where SNR is the signal-to-noise ratio) as a function of $r_{1}$ and $r_{2}$ with the postprocessing and in Fig. 1(c) the $\mathrm{SNR}_{\text {out }}$ without the postprocessing. Both were calculated using a spoke target as the scene. We observe that two configurations lead to local maxima. The first one is a two-ring BPM with $r_{1}=0.8$. It leads to an $\mathrm{SNR}_{\text {out }}$ of $12.6 \mathrm{~dB}$ without postprocessing and $19 \mathrm{~dB}$ with postprocessing. This shows, in particular, the benefit of postprocessing on the image quality. The second local maximum 
(b) $\mathrm{SNR}_{\text {out }}$ with post-processing

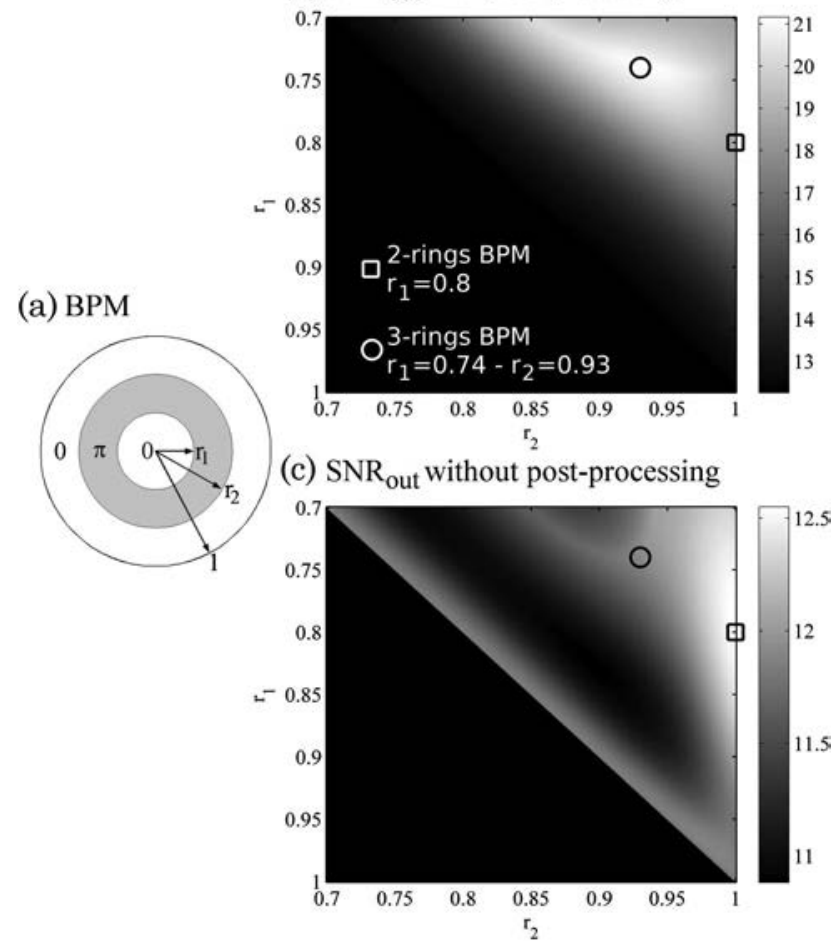

Fig. 1. (a) BPM structure and $\mathrm{SNR}_{\text {out }}(\mathrm{dB})$ of the imaging system according to the BPM parameters (b) with and (c) without postprocessing and optimal BPM parameters.

is a three-ring BPM with $r_{1}=0.74$ and $r_{2}=0.93$, leading to an $\mathrm{SNR}_{\text {out }}$ of $11.9 \mathrm{~dB}$ without postprocessing and $21 \mathrm{~dB}$ with postprocessing. We chose to implement a two-ring BPM since it is easier to manufacture, more tolerant to fabrication errors, and more efficient without postprocessing.

For experimental validation, the optimal BPM has been manufactured using three different techniques: diamond turning of a germanium substrate, etching of a germanium substrate, and subwavelength structuration of a GaAs substrate (Fig. 2). The two former technologies are easy to operate. The latter is more difficult to handle but generally allows one to generate more complex masks. The phase excursion is performed by a periodic lattice of pillars of a period much smaller than the wavelength behaving as an artificial media with an effective index. The calculation of the effective index is performed with full-vector theory software [5] relying on the rigorous coupled-wave analysis [6].

All types of BPM were implemented onto the camera. The observed scene, described in Fig. 3, is composed of three perforated metal plates, located at three different distances from the camera, 51,70 , and $100 \mathrm{~cm}$. Ahead, a flat panel display (LCD) provides a uniform heat background. The camera focused at $70 \mathrm{~cm}$ provides a sharp image while plate 2 is set in the distance range $[62-80 \mathrm{~cm}]$, which corresponds to $\psi=[-2.5 ; 2.5]$. In such condition, plates 1 and 3 are out of focus as observed in Fig. 4(a). By introducing the BPM, the whole image is a bit blurred but plates 1 and 3 become more visible [Fig. 4(b)]. With the implementation of the postprocessing, all the plate images are sharp. It can be noticed that the holes of plate 3 and the text "THALES" above it are now perfectly visible as (a) Global view of the subwavelength BPM

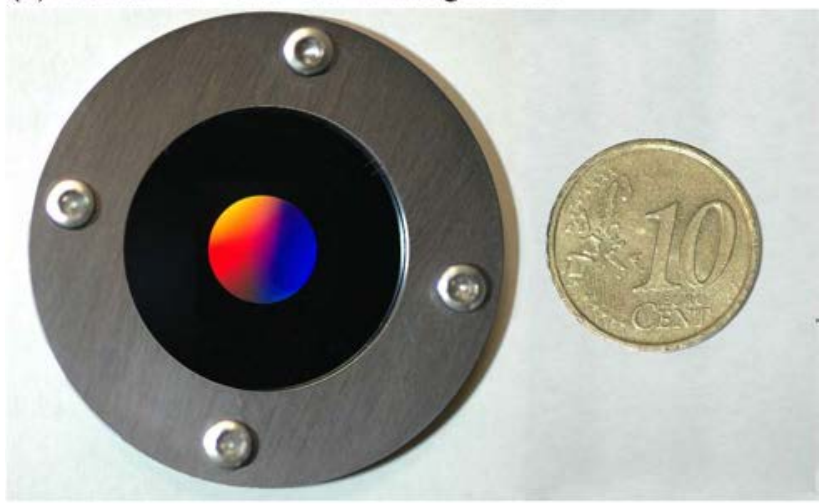

(b) Details of the BPM

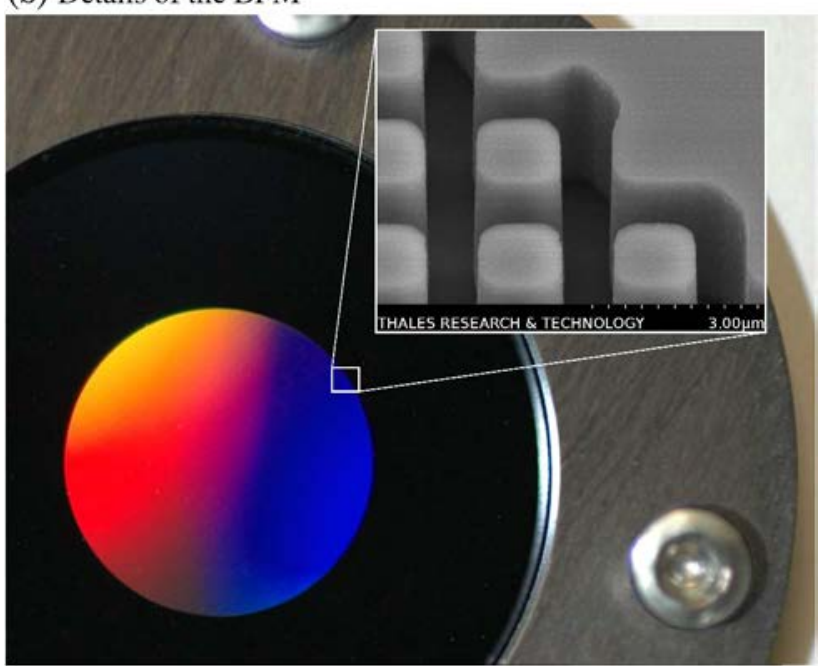

Fig. 2. (Color online) Subwavelength BPM and scanning electron microphotograph showing the border between the inner and the outer ring. To synthesize the desired effective index and thus the desired phase in the inner zone, the geometry of the pillars is controlled properly and manufactured through optical lithography and inductively coupled plasma etching.

well as the LCD trademark located a bit farther than plate 3 [Fig. 4(c)]. The depth of field has been effectively extended to $[51-112 \mathrm{~cm}]$, corresponding to $\psi=[-7.6 ; 7.6]$, reaching the expected improvement of a factor 3.

To compare the three mask fabrication technologies, we show in Figs. 4(d)-4(f) the postprocessed images of plate 3 obtained with each technology together with the obtained $\mathrm{SNR}_{\text {out }}$. The visual impression as well as the $\mathrm{SNR}_{\text {out }}$ are equivalent whatever the fabrication technology of the BPM.

Beside previous image quality consideration, we point out that such results are obtained in real time. For that

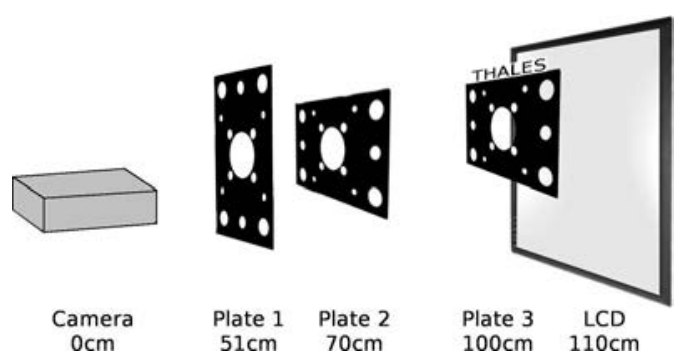

Fig. 3. Schematic of the experimental setup. 
(a) Without the BPM

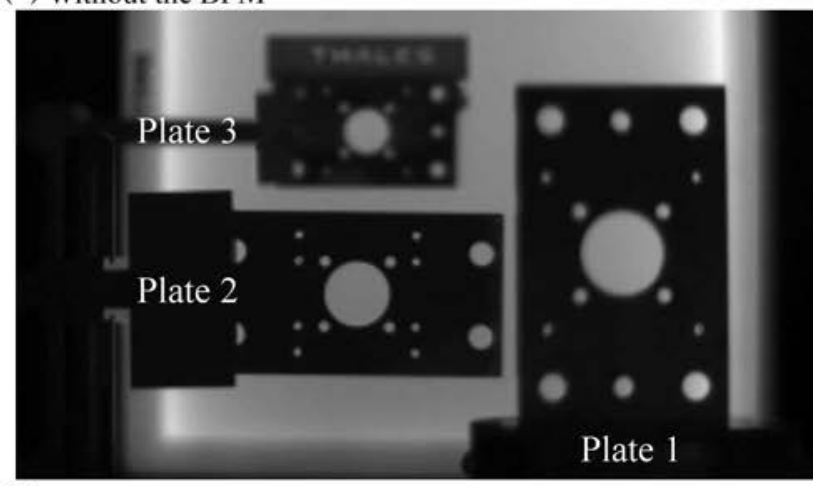

(b) With the BPM

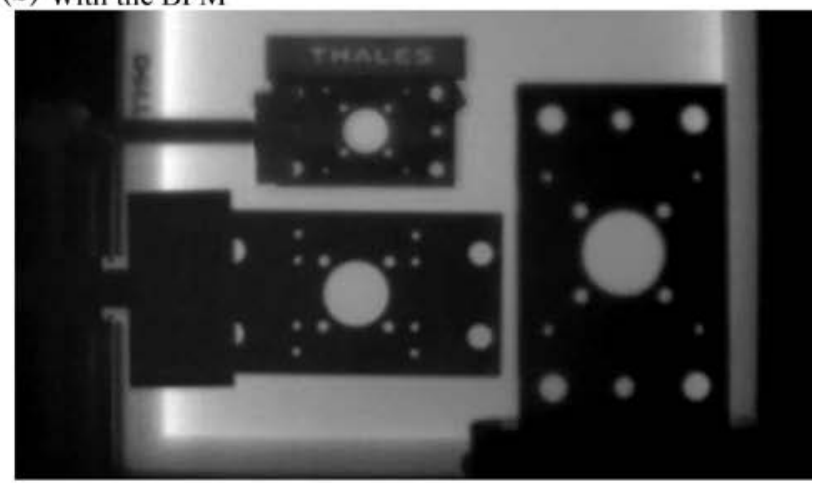

(c) With the BPM and the post-processing

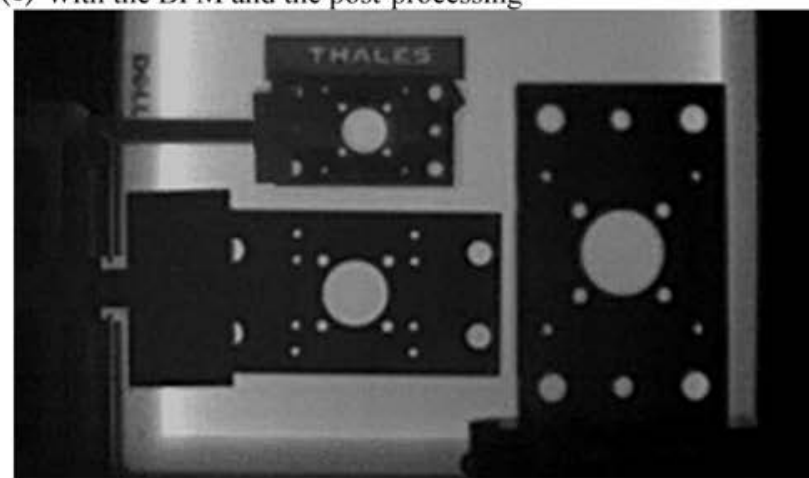

(d) Diamond $15.9 \mathrm{~dB}$

(e) Etching $16.3 \mathrm{~dB}$

(f) Subwavelength $16.2 \mathrm{~dB}$
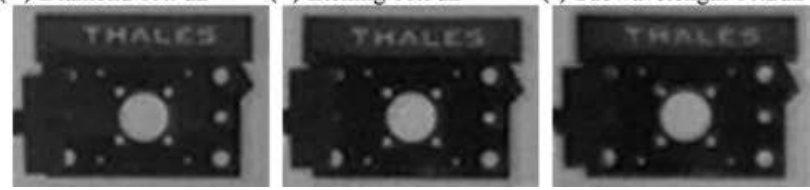

Fig. 4. Comparison of the image obtained (a) without the BPM, (b) with the BPM, and (c) with the postprocessing. (d)-(f) Details of the postprocessed images obtained with the different BPM.

purpose, several ways to perform the postprocessing were investigated. The first one consisted of calculating the deconvolution in the Fourier space with an off-theshelf computer graphics processing unit (GPU), which provides an easily accessible source of computational power. Such an approach enabled us to process an image with $512 \times 512$ pixels at 80 frames per second. The consumption is $30 \mathrm{~W}$ for the computer including the GPU. The second method consisted of using a programmable accelerator on field programmable gate array technology [7]. As this accelerator is a single instruction, multiple data architecture, it is well adapted to convolution computation in real space. Such architecture, composed of 128 elementary processing elements running at $200 \mathrm{MHz}$, will process the same image with $512 \times 512$ pixels at 100 frames per second at a lower consumption of $15 \mathrm{~W}$ compared to a GPU.

In conclusion, we implemented in an uncooled thermal camera an optimized two-ring binary-phase mask, which allows one, in combination with postprocessing, to extend the depth of field of by a factor 3. Such results, obtained to our knowledge for the first time with a postprocessing performed in real time, demonstrate the capability of wavefront coding implementation at low computing power. We considered a Wiener-like filter for deconvolution and the MSE as the image quality criterion. It is an interesting perspective to generalize this study to more advanced deconvolution techniques and more accurate image quality assessment methods [8].

In addition, we pointed out that the three different technologies investigated to manufacture the phase masks lead to similar results. These results fit well with simulation based on the use of an image quality criterion, which allows a cross optimization of the whole hybrid imaging system. Finally, these results indicate that the hybrid imaging approach may offer new solutions for enhancing performance of low cost security equipments.

The authors thank Jean-Pierre Huignard for scientific support related to hybrid imaging studies; François Leprêtre and Emmanuel Buhon for the realization of the diamond turned BPM and the mechanical mount; Samuel Beaussier and Fabrice Lemonnier for their contribution to the postprocessing implementation; and Didier Thenot, Stéphane Xavier, and Cédric Lyoret for their participation in the process of the subwavelength BPMs.

\section{References}

1. E. Dowski and T. Cathey, Appl. Opt. 34, 1859 (1995).

2. F. Diaz, F. Goudail, B. Loiseaux, and J.-P. Huignard, Opt. Lett. 34, 2970 (2009).

3. F. Diaz, F. Goudail, B. Loiseaux, and J.-P. Huignard, J. Opt. Soc. Am. A 27, 2123 (2010).

4. H. Wang and F. Gan, Appl. Opt. 40, 5658 (2001).

5. J. P. Hugonin and P. Lalanne, trademark of the Institut d'Optique (2005).

6. M. G. Moharam, J. Opt. Soc. Am. A 12, 1068 (1995).

7. P. Bonnot, F. Lemonnier, G. Edelin, G. Gaillat, O. Ruch, and P. Gauget, Proceedings on Design, Automation and Test in Europe (EDAA, 2008), p. 610.

8. Z. Wang, A. C. Bovik, H. R. Sheikh, and E. P. Simoncelli, IEEE Trans. Image Process. 13, 600 (2004). 